\title{
Designing Science and Technology Park of Tabriz with Organic Architecture Approach, Inspired by Ants' Nest Algorithm
}

\author{
Zahra Ghannadian ${ }^{1} \&$ Shahriyar Shaghagh ${ }^{1}$ \\ ${ }^{1}$ Department of Arts and Architecture, Shabestar Branch, Islamic Azad University of Shabestar, Iran \\ Correspondence:Zahra Ghannadian, Department of Arts and Architecture, Shabestar Branch, Islamic Azad \\ University of Shabestar, Iran.Tel: 90-553-018-0609 Fax: 98-4133-357-702. E-mail: Zohal.art@gmail.com
}

Received: June 26, 2016

doi:10.5539/mas.v11n1p134

\author{
Accepted: August 19, 2016 \\ Online Published: November 2, 2016 \\ URL: http://dx.doi.org/10.5539/mas.v11n1p134
}

\begin{abstract}
Establishment of every urban element in its appropriate position requires advanced technology and science from geographical and climatic point of view. This is accompanied and synchronous with modern culture born by supreme and high level of training and education in the society. In this regard, one of the main objectives of the architectures' union is to establish science and Technology Park with the aim of facilitating and accelerating science and technology transmission from university to the society and country.Tabriz city is considered as one of the most important academic and industrial cities in the country. Establishing science and technology parks can be effective in scientific, economic and social development of the country and province. Employing organic methods in architecture to use natural energies is an effective and useful method and its structure produces less pollution. Using philosophical and introductory issues and views of technology and establishing technology parks, present article proceeds on issues of organic architecture with new approach which is inspired by ants' nest algorithm and presents a new experience of architecture in the form of Science and Technology Park.
\end{abstract}

Keywords: science and technology park, organic architecture, ants' nest algorithm

\section{Introduction}

What can be understood of concept of technology in the modern era, is its mostly changed aspect i.e. dynamism of technology.Nowadays the technology passes language and race borders and its dynamism increases gradually. Learning new technologies, micro-chips and computer software take place faster than coal and iron technology. Technology parks and growth centers are established mainly close to industrial poles and areas' industry are established by the areas' university and technology units with the aim of developing technological communications and their main objective is to develop existing industries' technology at related industrial pole and to increase their competition power (Petree et al.,2000). Accumulation of technology units in these areas provides the conditions for more exploitation of the industries in addition to helping to improve and promote level of scientific and technical transactions among related units.Technology parks are ownership-based developments at a physical environment with higher quality and pseudo-park place (Asghari et al.,2010).

They enjoy advantages of closeness to the most important spiritual resources, appropriate infrastructures and leading policies, supporting technology-based companies and state corporations in a controlled environment, hence facilitating interaction and development of technology and economic growth. Providing physical and objective conditions needed for training and booming researchers and innovators through creating appropriate environments, bears saving in social time and communication costs through gathering a set of elements, entities, companies, organizations, and even individuals who are active in the field of creating, training, producing and improving quality of one or more defined technology needed in the society, facilitating in-person relationships and closeness of all elements which are active in the cycle of research, development and production, holding differentprofessional and educational seminars, conferences and meetings for scientific andtechnological development, economic savingsin investment of infrastructures, shared use of infrastructure facilities such as urban installations, preventing costly equipment of laboratories, data banks and , etc. optimal use of financial capitals of micro-companies and their professional scientific capabilities, elimination of obstacles on the way of development of such companies by providing infrastructural environments and facilities with fewer costs and encouraging them to work in the field of technologies which are needed in the society, providing implementation conditions for interdisciplinary studies, establishing environments with outstanding cultural and relationship 
characteristics, facilitation of planning and management for industries, accumulation of industries in a unique area, possibility ofmore accurate planning, better control of allocation of research budgets and evaluation of research organizations(Jalali et al.,2007).

\section{Concept of Technology Parks}

The main activities of research and development parks focus on high technology and undoubtedly they rely on researches which have been organized in line with knowledge-based development.Nowadays the world of science relies on inspiration by nature in its problem solution. The idea of designing such algorithms which is known as "meta-innovative algorithms" originate from different sources such as neurons, evolution process, models of social interactions among human beings and body immunity system. Meanwhile, "ants' nest algorithm" as problem solution technic is inspired by natural ants' behavior. The algorithm is a sub category of "mass intelligence" which acts based on pheromones amount discharged by the ant to solution parts and the algorithm obtains optimal answer through obtaining initial solution in each repetition. Considering that the aim of designing such complex is providing conditions needed for attracting experts, facilitation of having access to internet, e-books and electronic articles and displaying high tech in the form of completely organic environment inspired by ants' nest, so it seems necessary to establish an environment to meet above mentioned needs, exchange of thoughts and introducing high techs (Khaleghiyan, ).

Since there is no such environment not only in Tabriz city but also in Iran, individuals can not focus on new technologies and developments in this field will not have desired results in practice. Hence the balance betweensupply and demand will be vanished. The aim of presenting this design is to establish an interaction between above mentioned items which in turn results in solving the problems. The aspect of being organic helps us to establish a balance between underlying spaces and the nature and individuals of the environment will feel more relaxation.Moreover we will establish a relationship between technology and the nature through ants' nest algorithm which makes the design unique and reduces costs of installations in practice (Khaleghiyan, ).

A scientific park is an organization which is managed by professional experts and its main objective is to increase wealth in the society through improving and promoting innovation culture, competition among companies of the park and knowledge-based companies (Khaleghiyan, ). To achieve this goal, a scientific park motivates and manages science and technology movement among universities, research and development organizations, private companies and the market and doing so, it facilitates innovation-oriented companies through generative growth and processes centers.Technology parks, also, provide other high added-value services along with high quality workplace and facilities (Shaghaghi, 2009).

\section{Ants' Nest Algorithm}

Ant colony algorithm which is inspired by ants' behavior in finding path between nest and food was introduced for the first time by Marko Dorigo et.al in his $\mathrm{PhD}$ dissertation as a multi factor solution for optimization of problems such as itinerant vendor. The intelligent agent is a creature which can understand its surrounding environment by sensors and using influencers it can influence the environment. Ant colony algorithm was inspired by studies and observations on ants' colonies (Maleki, 2008). These studies have indicated that ants are collective insects which are living in colonies and they behave mostly for surviving whole colony rather than just one part of it. One of the most important behaviors of ants is their behavior for finding food, especially finding shortest path between food source and their nest. Such behavior of ants enjoys one kind of mass intelligence which has attracted scientists' attention recently. Ants leave a trace of pheromone chemical substance while moving. This substance, of course, evaporates but it remains as ant trace on the ground surface [9].

When they want to choose the path, they would prefer the one which has more pheromone, in other words they choose the path in which more ants have commuted. In its moving if ant chooses shortest and the most appropriate path between source node and target node, then mean distribution of pheromone will be more in the path. On the other hand if it chooses weak path, pheromone amount will be less in the path. In fact the amount of pheromone left in the path will be proportional with path quality. Doing so, the ants can choose shortest path in one graph. 


\section{Ants' Algorithm Analysis}

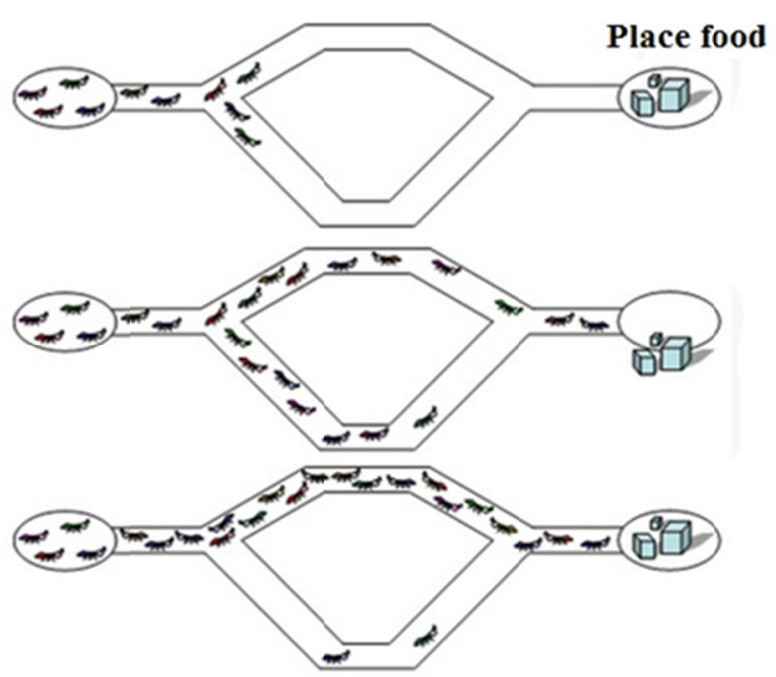

Figure 1. Ants' nest algorithm system

Ant system is a populated intelligent system, imitating ants' behavior of finding food which involves lots of factors with simple behaviors, being related to each other directly or indirectly. All agents are responsible for searching round paths and report path quality which is defined as the concept of "path performance".

So whenever the ant finds shortest (best) path from nest to food, it is more probable that remaining ants follow it at the same path. Gradually all ants will be at the same path with continuous improvement of the path and evaporating other paths. The aim of ants' algorithm is to imitate such behavior of ants which are moving on the diagram. The most important problem is to find shortest path and the solution for such problem is these ants (Mohammadi, 2009; Malekifar \& Rezaie, 2006).

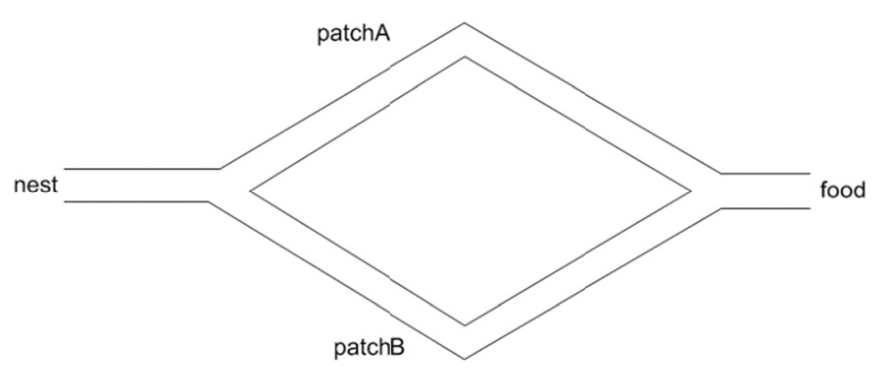

Figure 2.Scheme of investigation of random model

\section{Random Model}

Indirect relationship among ants in adjustability with the environment through leaving pheromone to influence other members' behavior: 


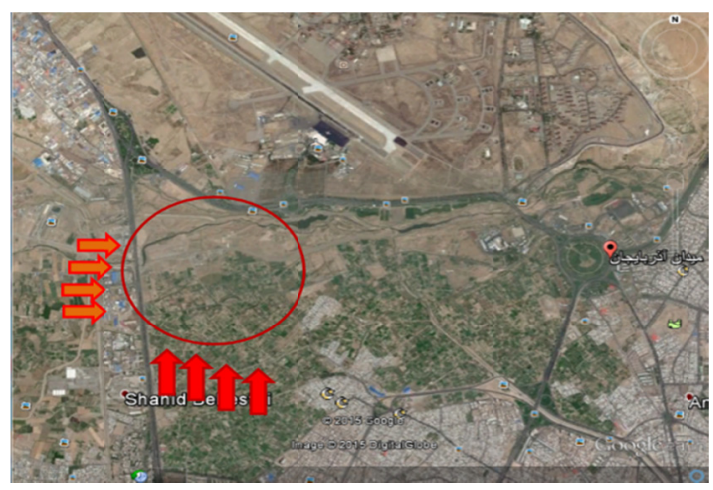

Figure3.Scheme of investigation of random model

The probability for choosing path A by next ant:(1)

$$
\rho_{A}(t+1)=\frac{\left(c+n_{A}(t)\right)^{\alpha}}{\left(c+n_{A}(t)\right)^{\alpha}+\left(c+n_{B}\right)^{\alpha}}=1-P_{B}(t+1)(1)
$$

Where $\mathrm{n}_{\mathrm{A}}(\mathrm{t})$ and $\mathrm{n}_{\mathrm{B}}(\mathrm{t})$ shows number of ants which move at time $\mathrm{t}$ in paths A and B.C shows absorption degree for unknown path and $\mathrm{C}$ shows more amount of pheromone for not choosing random path.

$\alpha$, shows bias toward pheromone left in the decision making process.

\section{Design Position and Area}

Investigation and studies carried out in Tabriz city in the fields of geographical and climatic features, give us a vision to determine its effect on the design by collecting data and to change them to practical and feasible guidelines. Cold climate and its relatively long duration in Tabriz city, makes it necessary to use sunlight and orient buildings design toward sunlight. Since Tabriz city is considered as highly risky area in zoning earthquake areas, it is necessary to consider following items in architecture and designing structure:

1) simplicity of plan and height

2) dividing irregular plans to simple plans

3) closeness of plan dimensions to each other

4) avoiding sudden change in height of plan's dimensions

5) Symmetry of plan

6) having mechanical balance and symmetry

\section{General Objectives Influencing the Design}

1) coping with coldness in half of the year

2) maximum use of solar energy in winter

3) reducing heat loss of the building by avoiding establishment of buildings at negative slopes and hollows

4) establishing buildings in areas which are back to the wind

5) preventing erosion due to raining and coldness by using durable materials in exterior cover of the building

6) designing compressed plans for buildings

7) creating filter spaces in entrances and minimizing changeability level of building's internal air in winter

8) optimal use of daily oscillation by employing heavy materials and integrated thermal insulations at external surfaces with delay time more than 8 hours

\section{AnalyzingSite Considered for Designing}

The project site has been located at proximity of Tabriz airport. It is bounded by Pilot Babayi Blvd. in the north, it is adjacent to Kargar Blvd. in the west and Azerbaijan Square in the East and it is adjacent to Santo road in the south. The site borders have been bounded by above mentioned streets and Azerbaijan Square has been located at the east part of the site. The site is in triangle form drawn from west to east. Accesses are Santoo road in the south and Kargar St. in the west. 


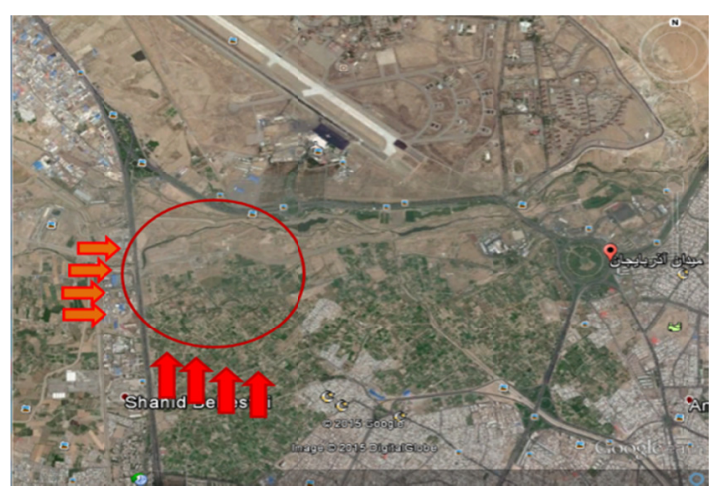

Figure 4. Investigation of position of designed site

The underlying site has been located almost at entrance of the city and it has the best position for establishing Science and Technology House. Due to adjacency to the main road (Santoo road), it has direct access to transportation network. Access is possible for all from southern and western angles. Among the reasons for choosing this area as design site are closeness to industrial towns, possibility of creating public places, appropriate designing and providing appropriate conditions for airport, having no steep slope and inappropriate typography, hills, mountains, floodway, factory and having easy access to parking place around the site.

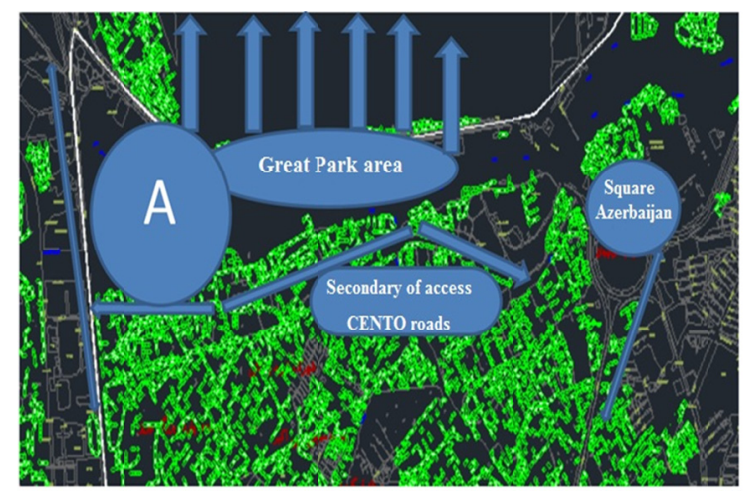

Figure 5. scheme of placement and access to the roads

\section{Formation Process of the Main Idea of the Project}

Using concepts of organic architecture, in design of the project we can achieve a completely organic design which is totally different from what has been seen earlier. General design of the complex was inspired by the concept of incubator. The main size of the project was inspired by ants' nest algorithm for optimal positioning.

The land position, paradise-like space of the park and street are connected to each other as two public urban places in a way that person finds himself at real size of the space through access and special communication patterns inspired by ants' nest algorithm. The conceptualism view of the designing of the complex was inspired by design strategy and idea. Mentality of growth is developed in the minds and it encourages human to move forward.

Placement of main size linearly, while convincing individuals' midstream, establishes one kind of relationship between technology park and its operational nature, leading the designer to achieve what has been formed in his mind. Openness of most lands of the complex which shows Iranian garden in the carpets, use of old principles of Iranian architecture,complex and building's geometrical integrity, simple and symbolic size and following principles of organicarchitecture have been always considered in the process of designing.

\section{Main Idea}

Open and wide vision as much as possible,dry and cold climate,designing science and technology house based on organic architecture,stretched site 


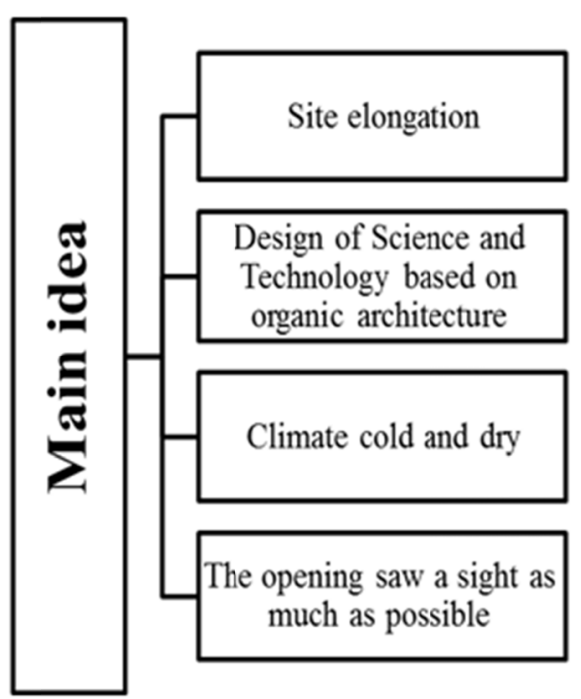

Figure 6.Site formation process

Considering concepts inspired by the site and characteristics of issues of ideogram, the project was designed based on parameters mentioned in Figure 6.
1) maximizing human presence in the life of technology
2) Giving authenticity to human movement
3) longitudinal expansion of the complex
4) creating dynamism in the site
5) flexiblity in the space

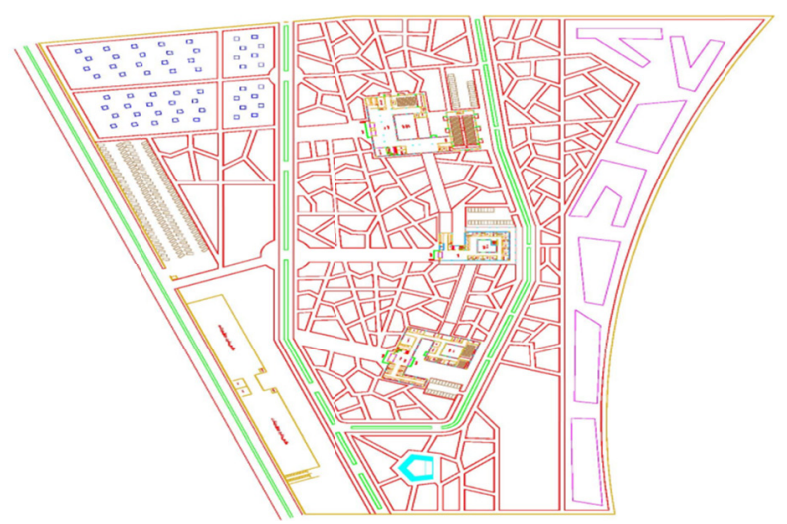

Figure 7.Design schema from above

With creating public spaces and providing appropriate conditions in the design in addition to placing individuals alongside each other, their privacy is also respected. Hence special spaces were considered in floors and in the site. 


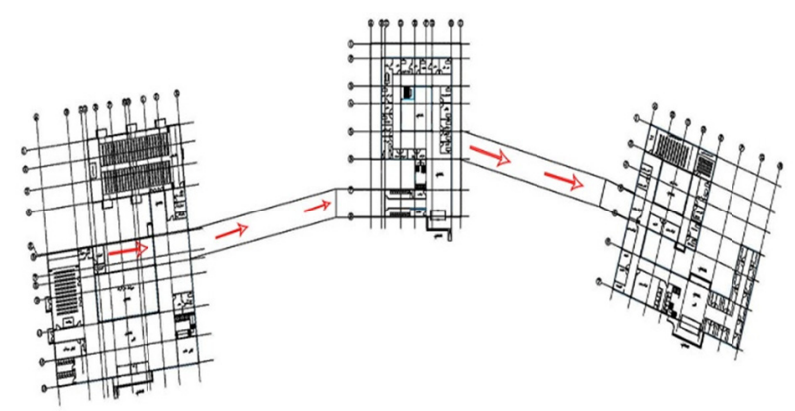

Figure 8.Orientation of site model with ants' algorithm

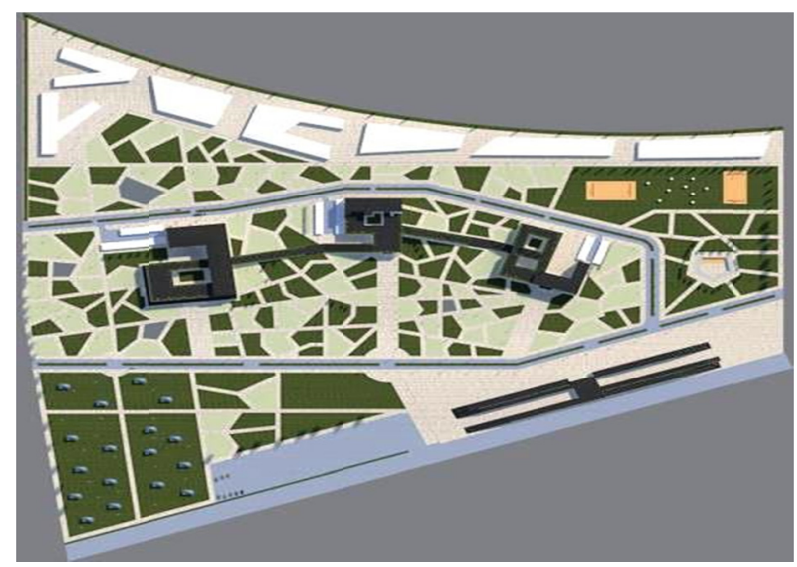

Figure 9. The completed site from above view

\section{Results}

In this study it was tried to make a connection between technology and organic architecture. Depending on conditions of achievement, new qualities can also be considered and to display it we can use materials, spacing methods, spatial fluidity, water, light, sky, going to the heart of earth or being on top of it which are characteristics of organic architecture. Science and Technology Park is in direct relationship with economy. Such centers can act as economic and income resource for people in the city and or all country at macro level. Designing science and technology parks based on solutions and algorithms of organic architecture and based on ants' nest algorithm can provide needed conditions for establishment of science and technology parks in Iran.

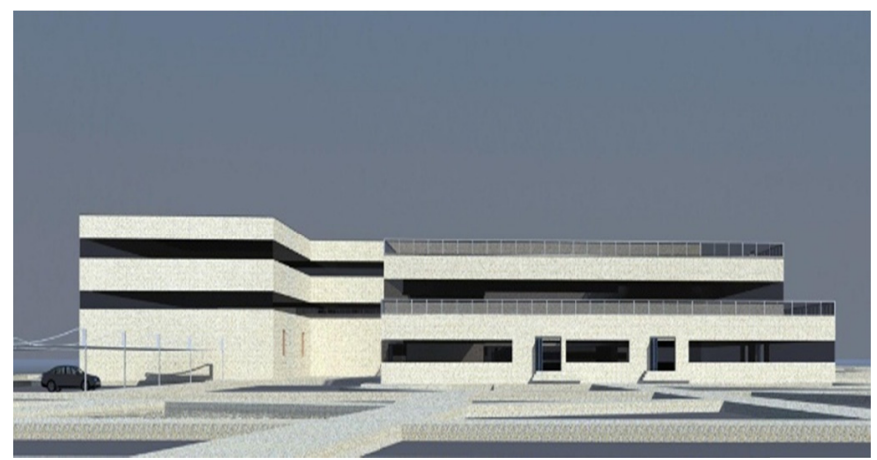

Figure 10. West view of designed building for science and Technology Park 


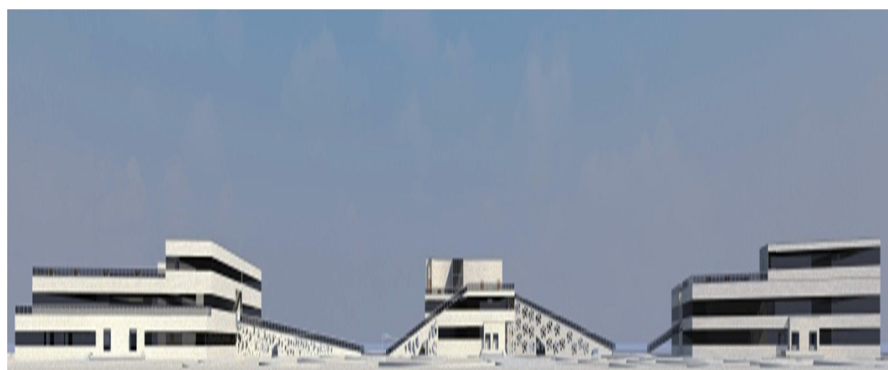

Figure 11. South view of designed building for science and Technology Park

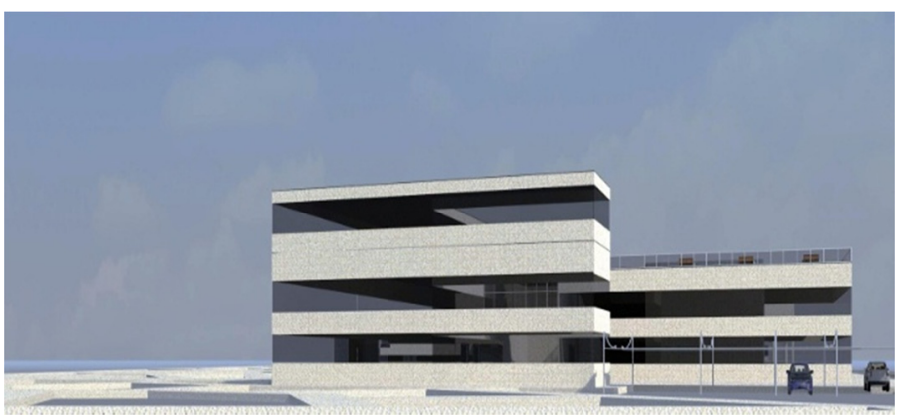

Figure 12.East view of designed building for science and Technology Park

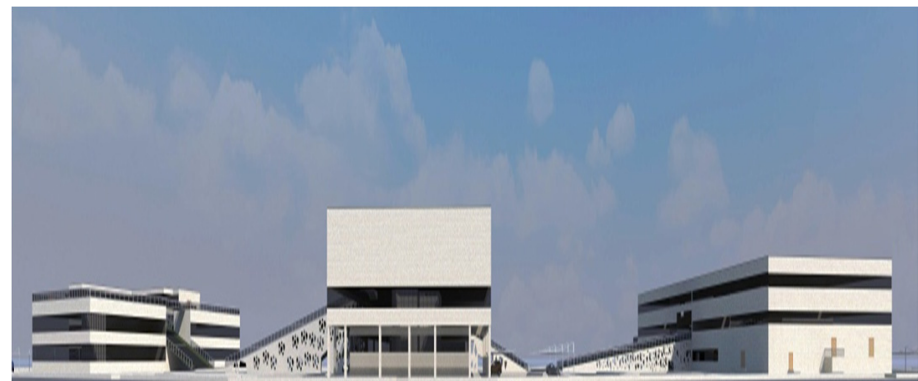

Figure 14.North view of designed building for science and Technology Park

\section{References}

Petree, R., Petkov, R., \& Spiro, E. (2000). Technology parks-concept and organization.Proceedings and reports of parks, technology and development centers, Tehran, Paradise Park.

Asghari, M.,Maasoumi, B., \&Meybodi, M. R. (2010).A new repelling ant colony based algorithm for improving the search and exploration power of rescue agents in rescue simulation system.

Jalali, N., Seyed, G., \&Ahmadizar, F. (2007).Optimization of reliability in series systems by multiple chooses and limited budget using ants' nest algorithm. Journal of Knowledge Management, 20(76),3-22.

Khaleghiyan, A. (2012).Legal structure and analysis of technology parks, proceedings and reports of parks, technology and development centers, Tehran, Paradise Park.

Khaleghiyan, A. (2013). Review of research parks in United States of America, proceedings and reports of parks, technology and development centers, Tehran, Paradise Park.

Khaleghiyan, A. (2014).Location of technology parks, Proceedings and reports of parks, technology and development centers, Tehran, Paradise Park (Rahimi Mahdi, Salehi, Ali, Naturalist (Organic) Architecture).

Shaghaghi, S.(2009).Studying and comparing characteristics of architecture and urban role of science and technology parks in Iran and in the world, Research Project, Islamic Azad University of Shabestar.

Maleki, M.(2008).Office space in Electronic era, M.A Thesis, Department of Architecture, Faculty of Architecture, Islamic Arts University of Tabriz, Tabriz. 
Mohammadi, E. (2009). Optimization of the problems with Ants' Colony Algorithm (ACO).Journal of Nazar, $3-4$.

Malekifar,A., \& Rezaie, H.(2006). Future technology and research scientific management in research centers, knowledge institutions, innovation factories. The article accepted in 3rd national congress of the state, University and Industry for national development. Study Center.

\section{Copyrights}

Copyright for this article is retained by the author(s), with first publication rights granted to the journal.

This is an open-access article distributed under the terms and conditions of the Creative Commons Attribution license (http://creativecommons.org/licenses/by/4.0/). 